Aloman 2020, 38(2), 69-82

Revista de Psicologia, Ciències de l'Eduació i de l'Esport

ISSN: $1138-3194$

CFacultat de Psicologia, Ciències de l'Educació i de l'Esport Blanquerna

Universitat Ramon Llull

\title{
RubricApp: Adaptación y Validación de una rúbrica para la Evaluación de Valor Pedagógico de las aplicaciones educativas móviles
}

\author{
Paola Pinilla-Morales ${ }^{1}$ \& María Graciela Badilla-Quintana ${ }^{2}$ \\ ${ }^{1}$ Facultad de Educación. Universidad Católica de la Santísima Concepción, Chile \\ ${ }^{2}$ Facultad de Educación. Universidad Católica de la Santísima Concepción, Chile \\ Centro de Investigación en Educación y Desarrollo CIEDE-UCSC \\ Universidad Católica de la Santísima Concepción, Chile
}

Recibido: 2020-06-02

Aceptado: 2020-10-29

RubricApp: Adaptación y Validación de una rúbrica para la Evaluación de Valor Pedagógico de las aplicaciones educativas móviles

Resumen. En la actualidad, la educación remota ha sido una gran oportunidad para garantizar la continuidad de los aprendizajes de los estudiantes y las tecnologías de la información y comunicación (TIC) se sitúan como la mejor opción para asegurarlos. Desde esta perspectiva, el aprendizaje móvil y las aplicaciones educativas han facilitado la tarea de muchos profesores que preparan clases a distancia. El desafío radica en la selección de recursos que cuenten con valor pedagógico y promuevan el aprendizaje esperado. Este artículo se propone como objetivos: a) reportar la adaptación y validación de una rúbrica que permita apreciar el valor educativo de las aplicaciones móviles, la cual logra un alfa de Cronbach, $\alpha=.783 ; b$ ) informar los resultados del estudio piloto, los cuales permiten contar con un instrumento denominado RubricApp, que se estructura en 10 indicadores y 3 dimensiones: valor pedagógico, diseño, y participación y motivación.

Palabras clave: rúbrica de evaluación, aplicaciones para móviles, fiabilidad

RubricApp: Adaptation and validation of a Rubric to evaluate the pedagogical value of Mobile Educational Applications

Summary. Nowadays, online education represents a tremendous opportunity for global education systems to guarantee the continuity of student learning, and Information and Communication Technologies (ICTs) must play a key role in addressing the challenges of contemporary education. From this perspective, mobile learning and educational apps have facilitated the tasks of many teachers that need to prepare virtual lessons. The challenge lies in the selection of resources that have pedagogical value and can help meet learning goals. The objective of this article is 1) to describe the adaptation and validation processes of a rubric designed to assess the educational value of mobile applications, and 2) to report the initial results of the application of this instrument. The resulting instrument is called RubricApp. It has a reliability coefficient of $\alpha=.783$ and is structured in 10 indicators and 3 dimensions: educational value, design, and participation and motivation.

Keywords: evaluation rubric; mobile apps; reliability

Correspondencia

Paola Pinilla-Morales

Alonso de Rivera 2850, Concepción

56-9-99098542

ppinilla@doctoradoedu.ucsc.cl 


\section{Introducción}

En la última década, los líderes educativos han asumido una visión integradora de las tecnologías de la información y comunicación (TIC) en sus centros educativos y, particularmente, en los procesos de aprendizaje (Fuentes et al., 2019). Estos recursos tecnológicos y digitales se han transformado en herramientas indispensables para el trabajo pedagógico del profesor, tanto fuera como dentro del aula. El interés por adquirir tecnología en las escuelas se incrementa (Cherner et al., 2016) para responder a las nuevas necesidades del aprendizaje de los estudiantes y de los contextos sociales emergentes. Asimismo, es habitual observar variados dispositivos tecnológicos en las aulas (Bonneton-Botté et al., 2020; Nikolopoulou, 2020) y mejores actitudes de los docentes para integrarlas al quehacer pedagógico (Ricoy y Sánchez, 2019; Salem y Mohammadzadeh, 2018; Semerci y Aydin, 2018; Zaranis y Oikonomidis, 2014).

En estudios referidos a la integración de TIC en la escuela y, en particular, cuando un docente las adopta en su quehacer educativo, se reconocen múltiples factores, tales como conocimiento, percepciones (AznarDíaz et al., 2019), creencias y prácticas (Ifinedo et al., 2019). Se advierten resultados favorables en el aprendizaje, por ejemplo, un aumento de la motivación y una mejor disposición al trabajo colaborativo (Stein et al., 2019), sin embargo, su uso no puede ser improvisado; si se desea conseguir efectos positivos en los aprendizajes de los estudiantes, imperiosamente debe ser planificado (Hernández et al., 2018).

Los últimos años, la integración de las tecnologías móviles se ha masificado, lo que se refleja en un mercado de aplicaciones móviles que se desarrolla aceleradamente. Así, se observa que, en el primer trimestre del 2020, los usuarios disponen de 2.56 millones de aplicaciones alojadas en Google Play por Android (el $96.3 \%$ son gratuitas), y 1.86 millones ofrecidas por el sistema operativo móvil de Apple Inc., iOS (el 90.84\% están liberadas de pago). Las aplicaciones de juego, educación, entretenimiento y fotografía tienden a ser las más descargadas; entre las más populares destacan los juegos y las aplicaciones educativas, seguidas por las de mensajerías y redes sociales (Statista, 2020). Khanna (2020) advierte que el 57\% del consumo de medios digitales de los usuarios está concentrado en el uso de aplicaciones móviles. Entre las diez aplicaciones más descargadas en el primer trimestre del 2020 lidera la aplicación TikTok, para crear vídeos cortos; seguido por WhatsApp, Facebook, Instagram, Messenger, Likee, SHAREit, Wall Picture, Snapchat y, en último lugar, YouTube (SensorTower, 2020). Una investigación con estudiantes de los cursos superiores de Primaria y hasta el segundo nivel de Bachillerato reporta que el uso que le otorgan a los dispositivos móviles con mayor frecuencia es para mensajería instantánea y redes sociales, casi siempre para escuchar música, usar la cámara de fotos, jugar a videojuegos, consumir películas y descargar aplicaciones; con menor frecuencia, para hacer videollamadas; rara vez, comprar en línea y realizar tareas administrativas (Besoli et al., 2018), que, sin duda, en los próximos años algunos de estos comportamientos, como uso de videollamadas, compras en línea y descarga de aplicaciones podrían invertirse en la escala de frecuencias y preferencias.

La irrupción de los dispositivos móviles en los espacios educativos (Lee y Cherner, 2015; Papadakis et al., 2017), la presencia de tabletas y las aplicaciones móviles (Gómez-Díaz et al., 2015) conlleva a la necesidad de replantearse el aprendizaje mediado por estas herramientas tecnológicas (Gil-Quintana, 2019) y, por ende, al surgimiento de un nuevo enfoque educativo denominado aprendizaje móvil o mobile learning (Torres Díaz et al., 2015). El aprendizaje móvil se entiende como una metodología de enseñanza y aprendizaje que se vale del uso de dispositivos móviles de pequeño tamaño, como, por ejemplo, teléfonos móviles, agendas electrónicas de bolsillo o PDA, tabletas, Pocket PC, iPod y todo dispositivo de mano que pueda ser conectado de forma inalámbrica (Santiago et al., 2015).

Sin embargo, los dispositivos móviles por sí solos no son capaces de generar aprendizajes, pues se entiende que son aparatos tecnológicos que requieren de un programa o software encargado de sostener los contenidos requeridos para que un estudiante pueda usar a favor de su aprendizaje. Cuando nos referimos al soporte de los contenidos de un dispositivo móvil, surge el concepto de aplicación móvil (o app). Las aplicaciones son softwares que se instalan en los dispositivos móviles, tales como teléfonos inteligentes (smartphones) y tabletas (tablets). "Ofrecen un recurso específico y acceso inmediato, sin la necesidad de entrar en internet. Por lo tanto, es posible descargarlas y acceder a ellas cuando y donde se desee, permiten desarrollar procesos de aprendizaje contextualizado" (Gillate et al., 2017, p. 117).

Sin duda, no basta el conocimiento para que un docente integre estas tecnologías a los procesos de enseñanza y aprendizaje de sus estudiantes, dada la amplia variedad de aplicaciones gratuitas y de bajo costo disponibles, es imprescindible evaluar su pertinencia educativa (Tondeur et al., 2016). En este sentido, se requiere de herramientas que le permitan distinguir si una aplicación que se define con fines educativos cumple criterios que demuestren su valor educativo. La literatura cuenta estudios sobre el diseño y la validación de rúbricas para evaluar las aplicaciones móviles y educativas (Buckler Peterson, 2012; Cherner et al., 2016; Lee y Cherner, 2015; Mize et al., 2019; Ok et al., 2015; Papadakis et al., 2017, que se transforman en una orientación confiable para que un profesor pueda emitir un juicio certero de la calidad de la aplicación móvil, sin embargo, la mayoría requieren de adaptaciones y validación, si desean aplicarse en contextos distintos.

La revisión sistemática de rúbricas para evaluar las aplicaciones educativas de Faizal-Yamimi et al. (2018) concluye que, la rúbrica integral para evaluar las aplicaciones móviles de Lee y Cherner reporta mayor efectividad para evaluar todo tipo de aplicaciones, a 
diferencia de otras, que se enfocan en aspectos específicos según los niveles, los grados o las poblaciones de sujetos (Faizal-Yamimi et al., 2018). Posterior a ese trabajo, Lee y Cherner, junto con otros investigadores, proponen una rúbrica a los profesores para evaluar las aplicaciones móviles, que permitiría observar su valor educativo (Cherner et al., 2016), con lo que se ofrecería a los docentes una herramienta orientadora para la selección de recursos tecnológicos idóneos para el aprendizaje.

En la actualidad, los profesores chilenos carecen de instrumentos validados para evaluar la idoneidad pedagógica o el valor educativo de una aplicación móvil, las únicas referencias disponibles para su selección son aquellas publicadas en páginas de internet y las que ofrecen las tiendas de descarga, que incluyen ficha de caracterización y calificación, y ésta última es asignada por los mismos usuarios. En este artículo se proponen dos objetivos: 1) Reportar la adaptación y la validación de una rúbrica por parte de expertos que permita apreciar el valor educativo de las aplicaciones móviles en el contexto del sistema escolar chileno y se caracterice por indicadores claros y precisos; con lo que se reduce el tiempo invertido para evaluar la aplicación móvil; 2) Informar de los resultados de la aplicación piloto del instrumento en dicho contexto. Para tales efectos, luego de una revisión de los instrumentos disponibles en la comunidad científica, se decide adaptar la rúbrica integral para evaluar aplicaciones educativas de Cherner et al. (2016).

\section{Diseño metodológico}

El primer objetivo señalado se aborda revisando las rúbricas para evaluar las aplicaciones móviles disponibles en la comunidad científica en las bases de datos académicas Web of Science (WOS) y Scopus, considerando como palabras clave los términos reconocidos en Unesco Thesaurus y el Thesaurus de Universidad de Barcelona (THUB): aplicaciones para móviles, aprendizaje móvil, evaluación y profesores, incluida la referencia del concepto en otra lengua como es el inglés, mobile app, applications, mobiles, evaluation, teachers. Se definen tres criterios de búsqueda: a) período de publicación:
2010-2020, b) categorías: educación, investigación educativa y, c) tipos de documentos: artículos científicos. De los resultados se obtienen 24 artículos en Web of Science y 23 artículos en Scopus, lo que suma un total de 47 artículos asociados a la búsqueda. Sin embargo, las investigaciones que arroja incluyen todo tipo de instrumentos de evaluación y en distintas especialidades, tales como salud y otras ciencias. Por tanto, se procede a refinar la búsqueda agregando el término rúbrica, que se encuentra presente entre las palabras clave de la mayoría de los estudios de evaluación de aplicaciones móviles que utilizan este tipo de instrumento y, el término educación, que circunscribe a dicha área. En esta etapa de búsqueda, los resultados que reportan los artículos científicos con tales criterios se reducen a 6 , los cuales se comparten en la tabla 1 .

Se decide adaptar la rúbrica de Cherner et al. (2016) considerando que los instrumentos disponibles en la literatura actual responden a criterios específicos, tales como nivel educativo (Papadakis et al., 2017), discapacidades o necesidades educativas especiales (Buckler y Peterson, 2012; Mize et al., 2019), disciplinas del saber o habilidades concretas (Gillate et al., 2017), entre otros, y este instrumento permitirá a los profesores evaluar aplicaciones para un espectro más amplio, tales como distintos niveles educativos y disciplinas de aprendizaje.

\section{Participantes}

El estudio del objetivo 1, asociado a la validez de contenido, se realizó con participantes expertos voluntarios (Angell, 2015; Escobar-Pérez y Cuervo-Martínez, 2008), por lo cual se invitó a especialistas que cumplieran con un perfil de experto TIC, y los criterios de selección fueron los siguientes: formación académica a nivel de posgrados y productividad científica con publicaciones durante los últimos cinco años en el área. Se contactó con el panel de expertos mediante correo electrónico, y quedó conformado por seis especialistas, cinco expertos chilenos y un experto internacional, a quienes se les envió el instrumento que había que validar y la matriz de valoración con las instrucciones para desarrollar el proceso.

Tabla 1. Resultados de la búsqueda de rúbricas para evaluar las aplicaciones móviles

\begin{tabular}{lll}
\hline Título & Autor / año & Revista / indexación \\
\hline $\begin{array}{l}\text { Developing a Rubric for evaluating reading } \\
\text { applications for learners with reading difficulties }\end{array}$ & $\begin{array}{l}\text { Mize, M., Park, Y., Schramm -Possinger, M. } \\
\text { y Coleman, M. (2019) }\end{array}$ & $\begin{array}{l}\text { Intervention in School and Clinic: SAGE Journals } \\
\text { Indexación Scopus }\end{array}$ \\
\hline $\begin{array}{l}\text { Designing and creating an educational app rubric } \\
\text { for preschool teachers }\end{array}$ & $\begin{array}{l}\text { Papadakis, S., Kalogiannakis, M. y Zaranis, N. } \\
(2017)\end{array}$ & $\begin{array}{l}\text { Education and Information Technologies } \\
\text { Indexación WOS - Scopus }\end{array}$ \\
\hline $\begin{array}{l}\text { A learning theory rubric for evaluating mobile } \\
\text { learning activities }\end{array}$ & Parsons, D. y MacCallum, K. (2017) & $\begin{array}{l}\text { International Journal of Online Pedagogy } \\
\text { and Course Design } \\
\text { Indexación Scopus }\end{array}$ \\
$\begin{array}{lll}\text { How to Find Good Apps: An Evaluation Rubric } \\
\text { for Instructional Apps for Teaching Students with } \\
\text { Learning Disabilities }\end{array}$ & $\begin{array}{l}\text { Ok, M.W., Kim, M.K., Kang, E.Y. y Bryant, B.R. } \\
(2016)\end{array}$ & $\begin{array}{l}\text { Intervention in School and Clinic } \\
\text { Indexación WOS - Scopus }\end{array}$ \\
$\begin{array}{l}\text { A detailed rubric for assessing the quality of } \\
\text { teacher resource apps }\end{array}$ & $\begin{array}{l}\text { Cherner, T., Lee, C.-Y., Fegely, A. y Santaniello, L. } \\
(2016)\end{array}$ & $\begin{array}{l}\text { Journal of Information Technology Education: } \\
\text { Innovations in Practice } \\
\text { Indexación Scopus }\end{array}$ \\
\hline $\begin{array}{l}\text { Developing an App Evaluation Rubric for } \\
\text { Practitioners in Special Education }\end{array}$ & Weng, P. L. (2015) & $\begin{array}{l}\text { Journal of Special Education Technology } \\
\text { Indexación Scopus }\end{array}$ \\
\hline $\begin{array}{l}\text { Nota. Elaboración propia. } \\
\text { Ind }\end{array}$ & &
\end{tabular}


El estudio del objetivo 2 se llevó a cabo invitando a docentes a utilizar el instrumento RubricApp para evaluar aplicaciones móviles para aprender a leer. Los profesores cumplieron con los criterios de ser educadores de nivel Preescolar o profesores de Primaria y que, atendieran a estudiantes de dichos niveles de enseñanza. Firmaron previamente el consentimiento informado de su participación en el estudio según las normas del Comité de Ética de la Universidad Católica de la Santísima Concepción de Chile. Se contó con la participación de 31 profesores, de los cuales 14 eran profesores de Primaria y 18, educadores de Preescolar, todos trabajando en aula en la actualidad y atendiendo a sus estudiantes de manera remota debido a la suspensión de clases por medidas sanitarias del país afectado por COVID-19.

\section{Instrumento}

Se realizó una adaptación de la rúbrica integral para evaluar las aplicaciones educativas de Cherner et al. (2016); vale destacar que, para efectos de esta investigación se contó con la autorización de los autores del instrumento. Una vez confirmado el permiso para adaptar la rúbrica se tradujo al español, con apoyo de una traductora de inglés a español, y se ajustó a la realidad del contexto educativo chileno. En su versión original, la rúbrica disponía de 3 dominios con 24 dimensiones (ver tabla 2).

Se revisó y se analizó cada uno de los dominios del instrumento a partir de los diferentes trabajos publicados que reportaban diseño, validación o uso de rúbricas

Tabla 2. Estructura de la rúbrica integral para evaluar aplicaciones móviles

\begin{tabular}{ll}
\hline Dominio & Dimensión \\
\hline Instrucción & A.1 Rigor \\
\hline & A.2 Habilidades del siglo xxi \\
\hline A.3 Conexiones con aprendizajes futuros \\
\hline A.4 Valor del error \\
\hline A.5 Retroalimentación del profesor \\
\hline A.6 Nivel de los contenidos \\
\hline A.7 Aprendizaje cooperativo \\
\hline A.8 Atención a las diferencias individuales \\
\hline B.1 Capacidad de guardar el proceso \\
\hline B.2 Integración \\
\hline B.3 Diseño de la pantalla \\
\hline B.4 Facilidad de uso \\
\hline B.5 Navegación \\
\hline B.6 Orientación de los objetivos \\
\hline B.7 Presentación de la información \\
\hline B.8 Integración de los medios \\
\hline B.9 Sensibilidad cultural \\
\hline C.1 Control del alumno \\
\hline C.2 Interactividad \\
\hline C.3 Ritmo \\
\hline C.4 Flexibilidad \\
\hline C.5 Interés \\
\hline C.6 Estética \\
\hline C.7 Utilidad \\
\hline 24 dimensiones \\
\hline
\end{tabular}

para evaluar aplicaciones móviles o educativas (Mize et al., 2019; Ok et al., 2016; Papadakis et al., 2017; Parsons y MacCallum, 2017; Weng, 2015), con la finalidad de encontrar elementos comunes. Es así como se llegó a la primera versión de RubricApp, una rúbrica analítica que cuenta con criterios definidos como indicadores, cuyo nivel de desempeño se presenta en descriptores graduados en escala de logro (Gonzaga, 2015). Si bien, la rúbrica de Cherner et al. (2016) se define como una rúbrica integral, también denominada holística, es decir, que por sus características se traduce en una evaluación global de las cualidades de una aplicación móvil, a nuestro juicio, fue necesario invitar a los docentes a una evaluación de los elementos y las características específicas de la aplicación móvil, acción que facilitó la valoración del evaluador (Badia, 2019; Panadero y Jonsson,2020), especialmente cuando no dominaba los aspectos técnicos del objeto que había que evaluar y establecer con detalle los niveles de logro que le otorgara mayor fiabilidad al instrumento (Jescovitch, 2019; Panadero y Jonsson, 2020). Por tales razones se estimaron las adaptaciones señaladas y específicamente, la modificación de su propósito, transitando de una rúbrica integral a una analítica.

Los ajustes que se aplicaron a la rúbrica de referencia incluían conservar los 3 dominios propuestos por los autores, los cuales dejaron de denominarse dominios y se propusieron como dimensiones: a) Valor pedagógico, b Diseño y c) Participación y motivación. Otro ajuste realizado en la versión original de la rúbrica estuvo relacionado con la reducción de indicadores: se eliminaron 9 por su baja relevancia para este estudio, otros 2 se unieron en 1 indicador dada su estrecha relación conceptual. De este modo, se logró disminuir la extensión del instrumento y reducir los tiempos invertidos en la evaluación de las aplicaciones móviles. La tabla 3 representa los ajustes realizados en la rúbrica integral (Cherner et al., 2016) y la estructura de RubricApp.

Los niveles de valoración del indicador se presentaron en descriptores tipo escala Likert de 5 puntos para favorecer la cuantificación (Cherner et al., 2016; GarcíaSanz, 2014; Lee y Cherner, 2015) de la valoración final de RubricApp, lo que permitió determinar si la aplicación móvil era recomendable para su uso con fines educativos. La rúbrica contó con un total de 70 puntos, cuyas categorías de valor fueron desde Muy bajo valor educativo a Muy alto valor educativo. Así, una aplicación móvil estaría en la categoría de Muy bajo, cuando lograra un puntaje en el rango de 1-14 puntos; Bajo, entre 15-28 puntos; Medio, entre 29-42 puntos; Alto, entre 43-56 puntos y, finalmente, Muy alto, entre 57-70 puntos.

\section{Validación de instrumento}

El proceso de validación de contenido por parte de los expertos se desarrolló utilizando una matriz de valoración, que se organizó en tres momentos y que incluía las instrucciones para desarrollar el proceso. El instru- 
Tabla 3. Ajustes de la rúbrica integral y estructura de RubricApp

\begin{tabular}{|c|c|c|c|c|c|}
\hline \multicolumn{3}{|c|}{ Rúbrica integral de Cherner et al. (2016) } & \multicolumn{3}{|l|}{ RubricApp } \\
\hline \multirow{2}{*}{$\frac{\text { Dominio }}{\text { Instrucción }}$} & \multicolumn{2}{|c|}{ Dimensión } & \multirow{9}{*}{$\begin{array}{l}\text { Dimensión } \\
\text { Valor pedagógico }\end{array}$} & \multirow{2}{*}{\multicolumn{2}{|c|}{$\begin{array}{l}\text { Indicadores } \\
\text { Se elimina } \\
\end{array}$}} \\
\hline & A.1 & Rigor & & & \\
\hline & $\overline{\mathrm{A} .2}$ & Habilidades del siglo xxI & & A.2. & Habilidades que estimula la aplicación \\
\hline & A.3 & Conexiones con aprendizajes futuros & & \multicolumn{2}{|c|}{ Se elimina } \\
\hline & A.4 & Valor del error & & A.4 & Valor del error \\
\hline & A.5 & Retroalimentación del profesor & & A.3. & Retroalimentación \\
\hline & A.6. & Nivel del material & & A.1 & Contenido \\
\hline & A.7 & Aprendizaje cooperativo & & A.5. & Aprendizaje cooperativo \\
\hline & A.8 & Atención a las diferencias individuales & & A.6. & Atención a las diferencias individuales \\
\hline \multirow[t]{9}{*}{ Diseño } & B.1 & Capacidad de guardar el progreso & \multirow[t]{9}{*}{ Diseño } & $\mathrm{B} 1$ & Capacidad para guardar el progreso \\
\hline & B.2. & Integración & & \multicolumn{2}{|c|}{ Se elimina } \\
\hline & B.3 & Diseño de la pantalla & & $\mathrm{B} 2$ & Diseño de la pantalla \\
\hline & $\underline{B} .4$ & Facilidad de uso & & \multirow[t]{2}{*}{ B3 } & \multirow[t]{2}{*}{ Facilidad de uso y navegación } \\
\hline & B.5 & Navegación & & & \\
\hline & B.6 & Orientación a los objetivos & & \multicolumn{2}{|c|}{ Se elimina } \\
\hline & B.7 & Presentación de la información & & $\underline{\mathrm{B} 4}$ & Presentación de la información \\
\hline & B.8 & Medios de integración & & \multicolumn{2}{|c|}{ Se elimina } \\
\hline & B.9 & Sensibilidad cultural & & \multicolumn{2}{|c|}{ Se elimina } \\
\hline \multirow[t]{7}{*}{ Compromiso } & C.1 & Control del alumno & \multirow{7}{*}{$\begin{array}{l}\text { Participación } \\
\text { y motivación }\end{array}$} & C.1. & Control de la aplicación \\
\hline & C. 2 & Interactividad & & \multicolumn{2}{|c|}{ Se elimina } \\
\hline & C.3 & Ritmo & & C.2 & Control de la velocidad \\
\hline & C. 4 & Flexibilidad & & \multicolumn{2}{|c|}{ Se elimina } \\
\hline & C. 5 & Interés & & C.3 & Interés \\
\hline & C.6 & Estética & & C.4 & Estética \\
\hline & C.7 & Utilidad & & \multicolumn{2}{|c|}{ Se elimina } \\
\hline Total: 3 dominios & \multicolumn{2}{|c|}{24 dimensiones } & 3 dimensiones & \multicolumn{2}{|c|}{14 indicadores } \\
\hline
\end{tabular}

Tabla 4. Momento 1. Validación de contenido por parte de los expertos

DIMENSIÓN A. Valor Pedagógico: las siguientes dimensiones se utilizan para medir el valor pedagógico de una aplicación. Indicador
A1. Contenido de la aplicación. ¿Los contenidos de la aplicación son apropiados para su público objetivo?

\begin{tabular}{|c|c|c|c|c|c|}
\hline 5 & 4 & 3 & 2 & 1 & Observación \\
\hline $\begin{array}{l}\text { El contenido de la } \\
\text { aplicación siempre es } \\
\text { adecuado para las edades } \\
\text { y los niveles educativos } \\
\text { especificados como } \\
\text { público objetivo. }\end{array}$ & $\begin{array}{l}\text { El contenido de la } \\
\text { aplicación } \\
\text { generalmente es } \\
\text { adecuado para las } \\
\text { edades y los niveles } \\
\text { educativos } \\
\text { especificados como } \\
\text { público objetivo. }\end{array}$ & $\begin{array}{l}\text { El contenido de la } \\
\text { aplicación rara vez } \\
\text { coincide con las edades } \\
\text { o los niveles } \\
\text { educativos } \\
\text { especificados como } \\
\text { público objetivo. }\end{array}$ & $\begin{array}{l}\text { El contenido de la } \\
\text { aplicación no se } \\
\text { corresponde con las } \\
\text { edades o los niveles } \\
\text { educativos } \\
\text { especificados como } \\
\text { público objetivo }\end{array}$ & $\begin{array}{l}\text { El contenido de la } \\
\text { aplicación es } \\
\text { inapropiado para fines } \\
\text { educativos. }\end{array}$ & \\
\hline \multicolumn{6}{|c|}{ ESPACIO PARA EL JUICIO DEL EXPERTO: } \\
\hline Criterios & ACEPTADA & NO ACEPTADA & \multicolumn{3}{|c|}{ OBSERVACIONES DEL EXPERTO } \\
\hline \multicolumn{6}{|l|}{ 1. Claridad del descriptor. } \\
\hline \multicolumn{6}{|l|}{ 2. Redacción adecuada. } \\
\hline \multicolumn{6}{|l|}{$\begin{array}{l}\text { 3. Progresión de la } \\
\text { valoración positiva o } \\
\text { negativa de fácil } \\
\text { apreciación. } \\
\end{array}$} \\
\hline \multicolumn{6}{|l|}{$\begin{array}{l}\text { 4. Criterios de valoración } \\
\text { (puntos } 1,2,3,4 ; 5 \text { ) } \\
\text { coherentes con la } \\
\text { graduación del } \\
\text { descriptor. } \\
\end{array}$} \\
\hline $\begin{array}{l}\text { 5. Descriptor coherente co } \\
\text { la dimensión. }\end{array}$ & & & & & \\
\hline
\end{tabular}

mento contó con afirmaciones y preguntas, que presentaban opciones de aceptación o rechazo, y que se valoraban con 1 y 2 respectivamente, y una columna adicional que permitía al experto agregar sus observaciones o comentarios. En el primer momento, el experto aceptaba o rechazaba los indicadores según criterios de claridad, redacción adecuada, progresión de la valoración, coherencia de la graduación y, por último, coherencia con la dimensión a la cual pertenecía. En la tabla 4, se presenta como ejemplo el primer indicador de la dimensión de valor pedagógico.

En el segundo momento, se le invitó a emitir un juicio global de toda la dimensión, donde el experto aceptaba o rechazaba según la coherencia de la totalidad de los indicadores con la dimensión y según si el valor de la dimensión se veía reflejado en los indica- 
Tabla 5. Momento 2. Validación de contenido por parte de los expertos

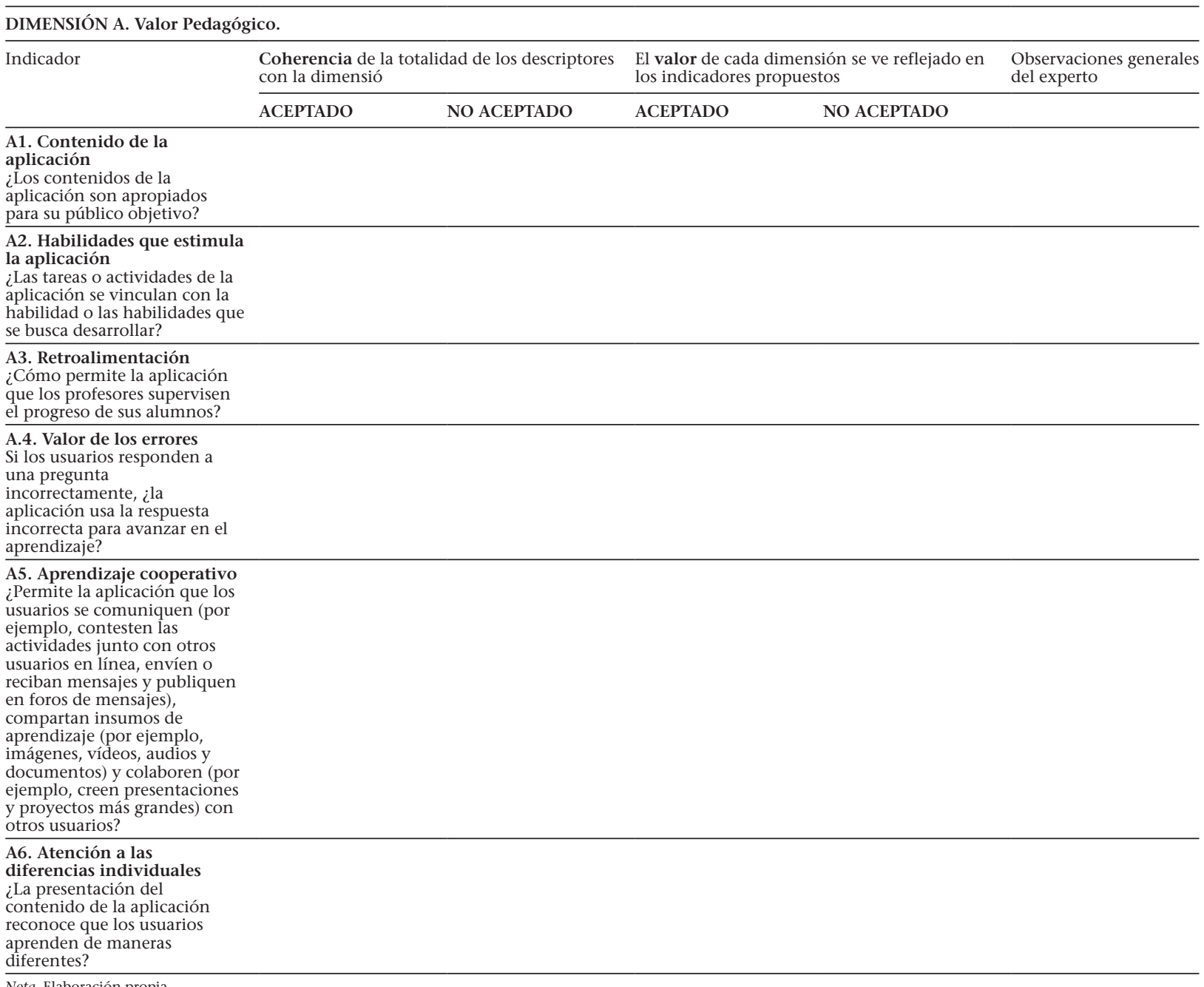

Nota. Elaboración propia.

dores propuestos. La tabla 5 representa el segundo momento de la validación por parte de los expertos.

En el tercer momento, se ofreció un espacio para incluir comentarios generales sobre el valor de la rúbrica analítica, para emitir un juicio relacionado con la idoneidad de una aplicación educativa para promover aprendizajes y ser usada en procesos educativos (ver tabla 6).

Los seis expertos que aceptaron participar en el proceso de validación de contenido enviaron la matriz con sus valoraciones de RubricApp por correo electrónico. Dichos datos se sistematizaron y se analizaron para realizar ajustes en el instrumento acorde a las sugerencias plasmadas en sus respuestas.

Tabla 6. Momento 3. Validación de contenido por parte de los expertos

Comentarios generales del valor de la rúbrica analítica para emitir un juicio con respecto a la idoneidad de una aplicación educativa para promover el aprendizaje y ser usada en procesos de enseñanza aprendizaje.

(registre aquí sus comentarios)

\section{Resultados}

Los resultados de dicho proceso dieron cuenta de que la mayoría de los indicadores de la dimensión A, valor pedagógico, cumplieron con los criterios observados, y solo los indicadores A.5. Aprendizaje cooperativo y A.6. Atención a las diferencias individuales lograron el 83\% de acuerdo entre los jueces; solo fueron rechazados por un experto que sugirió mejorar la redacción y acotar los elementos que debían ser observados en el momento de evaluar una aplicación móvil. Las siguientes dimensiones lograron un $100 \%$ de acuerdo entre los jueces en cada uno de sus indicadores y en su totalidad. Los expertos reconocieron la coherencia con la dimensión a la que pertenecían, por lo tanto, los indicadores de la dimensión A favorecían la observación del valor pedagógico de la aplicación móvil.

Los indicadores de la dimensión B, diseño, logró un $100 \%$ de acuerdo entre los jueces en relación con cada uno de los criterios observados, por lo tanto, se concluyó que permitía evaluar el diseño de una aplicación móvil para lograr el aprendizaje.

Por último, los indicadores de la dimensión C, participación y motivación, obtuvo el 100\% de acuerdo entre los expertos y se confirmó que sus indicadores 
permitían observar si la aplicación favorecía la participación y la motivación de los usuarios cuando interactuaban con la aplicación móvil.

Los resultados del segundo momento de evaluación de RubricApp en que los expertos emitieron un juicio global de la dimensión en su totalidad indicaron que, con relación a la dimensión A, valor pedagógico, el 100\% estuvo de acuerdo en la coherencia de los indicadores con la dimensión, y un $83 \%$ estuvo de acuerdo en la aceptación de todos los indicadores de la dimensión. Estos resultados se relacionaron con los del primer momento, donde se observó mejorar la redacción de algunos indicadores (A.5 y A.6) para asegurar la precisión de lo que se deseaba observar. Las dimensiones $\mathrm{B}$, diseño, y C, participación y motivación, lograron el 100\% de acuerdo en los dos criterios observados.

En el tercer momento, los jueces compartieron sus observaciones generales respecto al valor de RubricApp en la totalidad de los elementos de su estructura, específicamente se refirieron a si la aplicación educativa era idónea o apta para promover el aprendizaje y, por ende, si era recomendable su integración en procesos pedagógicos de enseñanza y aprendizaje. En esta instancia se recibieron observaciones de cuatro expertos, que indicaron que tanto las dimensiones como los indicadores eran apropiados para evaluar las aplicaciones móviles, y sugirieron atender a las observaciones de mejora de redacción. En la tabla 7 se observa el porcentaje de acuerdo y las sugerencias de los autores para mejorar el instrumento.

Las observaciones y los comentarios de los expertos permitieron enriquecer el diseño de RubricApp, estableciendo una estructura más robusta con 3 dimensiones y 14 indicadores que se apoyaban en una pregunta orientadora. La primera dimensión, que centró la observación en el valor pedagógico de la aplicación móvil se definió con 6 indicadores: contenido de la aplicación, habilidades que estimula la aplicación, retroalimentación, valor del error, aprendizaje colaborativo y atención a las diferencias individuales. La dimensión que se focalizó en observar el diseño de la aplicación, vale decir aspectos técnicos, contenía 4 indicadores que permitían observar la capacidad para guardar el progreso, el diseño de la pantalla, la facilidad de uso y la navegación, y la presentación de la información. Y, por último, la dimensión que observó las posibilidades de participación y motivación se organizaron en 4 indicadores: control del estudiante, control de la aplicación, interés y estética. La tabla 8 muestra la estructura de la versión final de RubricApp con la pregunta orientadora para cada indicador

Cada uno de los indicadores contó con descripciones progresivas de valoración, los valores fluctuaron de 5 a 1, donde el valor óptimo de logro se representó con 5 y el valor 1 dio cuenta de la ausencia de los criterios del descriptor (Cherner et al., 2016). Se dispuso, además, de un espacio para observaciones o comentarios que permitiera al evaluador registrar notas de aspectos que observara que pudieran ser significativos para las observaciones generales de la aplicación.

RubricApp logró una fiabilidad de $\alpha=$.783; con 10 ítems. La estimación estadística sugirió la eliminación de algunos indicadores que presentaban menor valor de correlación con el resto de los elementos del instrumento. En la tabla 9 se muestran los ajustes realizados en RubricApp.

Por lo tanto, una vez se hubieron realizado los análisis de confiabilidad de alfa de Cronbach se procedió a eliminar aquellos ítems que reportaban baja correlación con el resto de los elementos del instrumento y se finalizó el proceso de fiabilidad con 10 indicadores para observar el valor educativo de las aplicaciones móviles. Se modificó la distribución de puntajes que permitieran observar el valor educativo

Tabla 7. Resultados del juicio de los expertos

\begin{tabular}{|c|c|c|c|c|}
\hline & \multicolumn{2}{|c|}{ RubricApp } & \multicolumn{2}{|l|}{ Juicio de expertos } \\
\hline & & & Porcentaje de acuerdo & Observaciones \\
\hline \multirow[t]{6}{*}{ Valor pedagógico } & A.1 & Contenido & $100 \%$ & $\begin{array}{l}\text { Se acepta con mejoras de redacción: } \\
\text { contenido de la aplicación es idóneo para el } \\
\text { público al que está dirigido. }\end{array}$ \\
\hline & A. 2 & Habilidades que estimula la aplicación & $100 \%$ & $\begin{array}{l}\text { Se acepta con mejoras de redacción: observar } \\
\text { la vinculación de las tareas y actividades con } \\
\text { la habilidad que ofrece la aplicación. }\end{array}$ \\
\hline & A.3 & Retroalimentación & $100 \%$ & Se acepta. \\
\hline & A.4 & Valor del error & $100 \%$ & Se acepta. \\
\hline & A.5 & Dimensión & $83 \%$ & $\begin{array}{l}\text { Se acepta con mejoras de redacción: se } \\
\text { cambia el concepto cooperativo por el } \\
\text { colaborativo. }\end{array}$ \\
\hline & A.6 & Atención a las diferencias individuales & $83 \%$ & Se acepta. \\
\hline \multirow[t]{4}{*}{ Diseño } & $\underline{B}, 1$ & Capacidad para guardar el progreso & $100 \%$ & Se acepta. \\
\hline & B.2 & Diseño de la pantalla & $100 \%$ & Se acepta. \\
\hline & B.3 & Facilidad de uso y navegación & $100 \%$ & Se acepta. \\
\hline & B.4 & Presentación de la información & $100 \%$ & Se acepta. \\
\hline \multirow[t]{4}{*}{ Participación y motivación } & C.1 & Control de la aplicación & $100 \%$ & Se acepta. \\
\hline & C. 2 & Control de la velocidad & $100 \%$ & $\begin{array}{l}\text { Se acepta con mejoras de redacción: cambiar } \\
\text { el ritmo por el control de la velocidad. }\end{array}$ \\
\hline & C.3 & Interés & $100 \%$ & Se acepta. \\
\hline & C. 4 & Estética & $100 \%$ & Se acepta. \\
\hline 3 dimensiones & \multicolumn{2}{|c|}{14 indicadores } & \multicolumn{2}{|l|}{14 indicadores } \\
\hline
\end{tabular}


Tabla 8. Estructura final de RubricApp

\begin{tabular}{|c|c|c|c|}
\hline \multirow{2}{*}{$\frac{\text { Dimensión }}{\text { A. Valor pedagógico }}$} & \multicolumn{2}{|c|}{ Indicador } & \multirow{2}{*}{$\begin{array}{l}\text { Pregunta orientadora } \\
\text { ¿Los contenidos de la aplicación son apropiados para fines educativos? }\end{array}$} \\
\hline & A.1 & Contenido de la aplicación & \\
\hline & A. 2 & $\begin{array}{l}\text { Habilidades que estimula la aplicación } \\
\text { móvil }\end{array}$ & $\begin{array}{l}\text { ¿Las tareas o actividades de la aplicación desarrollan las habilidades que } \\
\text { ofrecen? }\end{array}$ \\
\hline & A.3 & Retroalimentación & $\begin{array}{l}\text { ¿La aplicación ofrece reporte de avance y logros obtenidos por el } \\
\text { estudiante? }\end{array}$ \\
\hline & A. 4 & Valor del error & $\begin{array}{l}\text { Si los estudiantes responden a una pregunta incorrectamente, ¿la } \\
\text { aplicación permite reconocer el error? }\end{array}$ \\
\hline & A.5 & Aprendizaje colaborativo & $\begin{array}{l}\text { ¿La aplicación permite que los estudiantes se comuniquen, compartan } \\
\text { insumos de aprendizaje y colaboren con otros usuarios? }\end{array}$ \\
\hline & A. 6 & Atención a las diferencias individuales & $\begin{array}{l}\text { ¿La aplicación presenta los contenidos en diversos formatos } \\
\text { reconociendo la diversidad de aprendizajes de los estudiantes? }\end{array}$ \\
\hline \multirow[t]{4}{*}{ B. Diseño } & B.1 & Capacidad para guardar el progreso & $\begin{array}{l}\text { ¿La aplicación permite que los estudiantes regresen al contenido que } \\
\text { estaban utilizando por última vez antes de cerrar la sesión? }\end{array}$ \\
\hline & B. 2 & Diseño de la pantalla & $\begin{array}{l}\text { ¿Los recursos audiovisuales de la aplicación (ejemplo: texto, gráficos, } \\
\text { vídeos, sonidos o discurso hablado) están bien organizados para el } \\
\text { desarrollo del contenido? }\end{array}$ \\
\hline & B. 3 & Facilidad de uso y navegación & $\begin{array}{l}\text { ¿El diseño de la aplicación permite al estudiante una interacción } \\
\text { intuitiva que logre que su ejecución sea por instrucción y orientación } \\
\text { mínima? }\end{array}$ \\
\hline & B. 4 & Presentación de la información & $\begin{array}{l}\text { ¿La aplicación presenta el contenido de manera lógica con niveles de } \\
\text { dificultad que estimulen el aprendizaje del estudiante? }\end{array}$ \\
\hline \multirow[t]{4}{*}{$\begin{array}{l}\text { C. Participación y } \\
\text { motivación }\end{array}$} & C.1 & Control de la aplicación & $\begin{array}{l}\text { ¿La aplicación permite a los usuarios seleccionar el nivel (por ejemplo, } \\
\text { nivel de grado, nivel de edad o nivel de dificultad) desde donde } \\
\text { comenzarán a navegar por el contenido? }\end{array}$ \\
\hline & C. 2 & Control de la velocidad & $\begin{array}{l}\text { ¿La aplicación permite a los usuarios controlar la velocidad de } \\
\text { interacción con el contenido? }\end{array}$ \\
\hline & C.3 & Interés & $\begin{array}{l}\text { ¿Es probable que el contenido de la aplicación atraiga a su público } \\
\text { objetivo? }\end{array}$ \\
\hline & C. 4 . & Estética & $\begin{array}{l}\text { ¿Los gráficos y la interfaz de la aplicación motivan a los usuarios a } \\
\text { interactuar? }\end{array}$ \\
\hline
\end{tabular}

Nota. Elaboración propia.

Tabla 9. Ajuste de la estructura de RubricApp

\begin{tabular}{ll}
\hline Sugerencias & $\alpha$ Cronbach \\
\hline Totalidad de indicadores de RubricApp & .692 \\
\hline Si elimina B.1. Capacidad para guardar el progreso & .749 \\
\hline Si elimina A.4. Valor de errores & .758 \\
\hline Si elimina A.6. Atención a las diferencias individuales & .773 \\
\hline Si elimina A.5. Aprendizaje colaborativo & .783 \\
\hline Nota. Elaboración propia. &
\end{tabular}

Tabla 10. Estructura final de RubricApp

\begin{tabular}{lll}
\hline Dimensión & \multicolumn{2}{l}{ Indicador } \\
\hline A. Valor pedagógico & A.1 & Contenido de la aplicación \\
\cline { 2 - 3 } & A.2 & $\begin{array}{l}\text { Habilidades que estimula } \\
\text { la aplicación móvil }\end{array}$ \\
\cline { 2 - 3 } & A.3 & Retroalimentación \\
\hline B. Diseño & B.1 & Diseño de la pantalla \\
\cline { 2 - 3 } & B.2 & Facilidad de uso y navegación \\
\hline C. Participación & B motivación & Presentación de la información \\
\cline { 2 - 2 } & C.2 & Control de la aplicación \\
\hline & C.3 & Interés \\
\hline & C.4. & Estética \\
\hline
\end{tabular}

Nota. Elaboración propia.

de la rúbrica, 1-10 puntos (muy bajo), 11-20 puntos (bajo), 21-30 puntos (medio), 31-40 puntos (alto), y 41-50 puntos (muy alto). En la tabla 10 se presenta la estructura final de RubricApp.

\section{Piloto de RubricApp}

Una vez finalizado el proceso de validación por parte de los expertos y obtenida el alfa de Cronbach, se pro- cedió a realizar un estudio preliminar de la aplicación del instrumento que permitió abordar el segundo objetivo de este estudio.

En primera instancia, las investigadoras utilizaban la rúbrica para evaluar aplicaciones móviles educativas disponibles en las tiendas virtuales. Se ingresó a la tienda Play Store y se seleccionaron aplicaciones que cumplieran con dos criterios: fines educativos y gratuidad. El procedimiento de búsqueda se realizó considerando el ingreso desde un teléfono móvil. Para acceder se registró, en la barra de buscar aplicaciones y juegos, las palabras clave aprender a leer. Se descargó la aplicación y se ejecutó a fin de usarla al mismo tiempo que se aplicaba RubricApp para indagar sobre su valor educativo.

A continuación, se presentan los resultados de la evaluación de tres aplicaciones móviles alojadas en Play Store, que permitieron observar las virtudes de RubricApp para determinar el valor educativo de una aplicación móvil según su valor pedagógico, diseño, participación y motivación.

La primera aplicación que arrojó la búsqueda en Play Store correspondió a Sílabas León, aprender a leer con sílabas, que en su descripción indicaba ser gratuita desde los 6 a 12 años, estaba enfocada a aprendizajes de letras y sílabas (Aprender Jugando, 2019). El resultado obtenido con RubricApp permitió observar que su valor educativo era alto, y logró 36 puntos en total. Esta aplicación consiguió su mejor puntaje en la dimensión diseño, dado que obtuvo 12 puntos de la rúbrica, lo que equivalía al $80 \%$ del valor total de dicha dimensión. El menor puntaje correspondió a la dimensión de participación y motivación, en la cual logró 13 
puntos, que representaban el $65 \%$ del valor total de la dimensión. La baja puntuación se debió al indicador del control de la aplicación, ya que su evaluación se situó en el nivel de progresión 2, lo que dio cuenta de que la aplicación educativa no permitía al estudiante escoger el nivel de dificultad y había que partir del nivel más bajo, pero sí permitía avanzar y retroceder.

La segunda aplicación se denominaba Aprender a leer, y estaba orientada al aprendizaje de sílabas y vocabulario (Carrion, 2019). Al aplicar RubricApp se observó un logro total de 26 puntos, por tanto, la situó como una aplicación con valor educativo medio. Sus limitaciones se visibilizaron en la dimensión de diseño, dado que obtuvo 4 puntos, con un porcentaje de logro equivalente al $26 \%$. Las dificultades de diseño se veían reflejadas en el indicador de diseño de la pantalla, además de en la facilidad de uso y navegación, las cuales obtuvieron el valor mínimo de 1 punto, por tanto, dio cuenta de que, en este indicador, podían acceder a la aplicación pero dependían de un apoyo permanente para navegar en esta por su complejidad en cuanto a la ruta de sus pantallas. La dimensión que obtuvo mejores resultados fue la de valor pedagógico, en la cual obtuvo 11 puntos, y permitió un 76\% de logro, lo cual dio cuenta de que los contenidos que ofrecía la aplicación eran apropiados para fines educativos. En cuanto a las habilidades, se observó que el 50\% de las actividades propuestas estaban vinculadas con las habilidades que declaraba abordar y en relación con la retroalimentación ofrecía al estudiante un reporte de sus avances y logros de las actividades realizadas, lo cual solo podía verse mientras se navegaba por la aplicación.

La tercera aplicación se denominó Leo con Grin, que en su descripción indicaba que era para niños de hasta 8 años de edad, y sus aprendizajes estaban centrados en la memorización visual y auditiva, la identificación y asociación, y la discriminación y comprensión de lectura (Educaplanet, 2019). Su evaluación con RubricApp reportó un puntaje total de 28 puntos, que indicaba un valor educativo medio. La dimensión mejor puntuada fue la de diseño, con 10 puntos, que representaban el 66\% de logro. La dimensión con mayores limitaciones en su evaluación correspondió a la dimensión de participación y motivación, con solo 9 puntos, que representaban el $45 \%$ de logro. Sus dificultades se concentraban en los indicadores C1. Control de la aplicación, C2. Velocidad y C3. Interés, en los que logró 2 puntos. El primer indicador dio cuenta de que la aplicación no permitía al estudiante escoger el nivel de dificultad, y debía partir del nivel más bajo, pero le permitía avanzar o retroceder dentro de la aplicación. En el segundo indicador, la aplicación no permitía controlar la velocidad del contenido, sin embargo, la velocidad era apropiada para navegar en la aplicación. Por último, el indicador de interés logró menos del 25\% que su contenido era atractivo y despertaba el interés de interacción y aprendizaje. Vale destacar, que esta aplicación solo era de su uso gratuito en una interfaz de seis que presentaba, cada interfaz desplegaba 5 letras, y en la primera interfaz, que permitía la navegación, el juego solo liberaba 2 letras, el resto de los contenidos eran de pago.

En resumen, de las tres aplicaciones sometidas a evaluación con RubricApp, solo la aplicación móvil Sílabas León, aprender a leer con sílabas (Aprender Jugando, 2019), logró un valor educativo alto. Las aplicaciones Aprender a leer (Carrion, 2019) y Leo con Grin (Educaplanet, 2019) reportaron bajas puntuaciones en algunas dimensiones, y se situaron en un valor educativo medio. Las fichas de análisis de las aplicaciones móviles mencionadas se encuentran en el anexo 1.

En una segunda instancia, se invitó a participar voluntariamente a 50 profesores de los niveles de Preescolar y Primaria para utilizar el instrumento obtenido en el proceso de validación. La invitación se extendió por medio de correo electrónico y se recibieron 32 respuestas Los sujetos, previa firma del consentimiento informado, participaron en un taller de capacitación en el que se les presentó RubricApp, las dimensiones y sus respectivos indicadores, y se aplicó la rúbrica en conjunto a una aplicación móvil con el fin de calibrar los juicios de cada evaluador.

Tras el taller, los profesionales evaluaron una aplicación móvil de su interés, cuyo criterio de contenido o habilidad vinculado con la lectoescritura se hubo acordado previamente. Los resultados reportaron el valor educativo de 12 aplicaciones disponibles en la tienda virtual de Android.

Los resultados del piloto indicaron que la aplicación Sílabas León, aprender a leer con sílabas (Aprender Jugando, 2019), fue la aplicación móvil que reportó más evaluaciones (20), y su valor educativo fue el de nivel alto, resultado que coincidió con el de la evaluación realizada en la primera fase del pilotaje. El valor educativo alto se logró con puntajes que oscilaron entre los 31 y los 40 puntos, no obstante, la tendencia de puntuaciones para esta aplicación se concentró entre los valores de 38 y 40 puntos. La dimensión con menos logros fue la dimensión C. participación y motivación, y el indicador C1. Control de la aplicación, el que reportó menor valoración.

La segunda aplicación móvil que concentró más valoraciones fue Aprender a leer (Carrion, 2019), dado que cinco evaluadores la escogieron para aplicar RubricApp. El valor educativo alcanzado fue de nivel medio, que coincidió con el resultado obtenido en la primera fase previa de este piloto. Este nivel de valor educativo se logró con puntuaciones de entre 21 y 30 puntos; no obstante, Aprender a leer, obtuvo resultados de entre 26 y 29 puntos, se concentra en las puntuaciones más altas del nivel.

Por último, vale destacar que la aplicación que logró el máximo nivel educativo que reportó RubricApp, es decir, el nivel muy alto, fue el obtenido por la aplicación móvil LetraKid Aprende a Escribir para niños ABC\&123, juego educativo infantil para niños de 4 a 8 años. A través de esta aplicación se aprende a escribir o a trazar las letras del abecedario español (ParKel Soft, 2020). El nivel educativo muy alto se obtuvo con puntuaciones que oscilaban entre los 41 y los 50 puntos; en el caso 
particular de esta aplicación móvil, los puntajes obtenidos fluctuaban entre los 42 y los 44 puntos, dado que, reportó menor logro en los indicadores B4. Presentación de la información y C1. Control de la aplicación.

\section{Discusión}

El primer objetivo del estudio fue adaptar y validar una rúbrica para que unos expertos evaluaran unas aplicaciones educativas y aseguraran que los procesos previos de adaptación idiomática y estructural de la rúbrica permitían contar con un instrumento que pudiera usarse en la selección de aplicaciones móviles para el aprendizaje, que además incluyó el cálculo de alfa de Cronbach para garantizar la fiabilidad del instrumento; y, el segundo objetivo propuesto definió pilotear $\mathrm{Ru}$ bricApp con profesores, ambos propósitos con el fin de contribuir con un instrumento validado y confiable apropiado para el contexto educativo chileno.

Las aplicaciones móviles se han transformado en una de las herramientas tecnológicas que, con mayor frecuencia, se han ido integrando en las salas de clases o en los procesos de aprendizaje (Cherner et al., 2016; Gómez Díaz et al., 2015; Kortabitarte et al., 2018) dentro o fuera del aula. El uso de dispositivos y aplicaciones móviles se ha masificado exponencialmente en la última década (Clement, 2020; Khanna, 2020; Statista, 2020) y los profesores se atreven a integrarlos en las actividades pedagógicas que proporcionan a sus estudiantes con mayor frecuencia. En tiempos de crisis sanitaria, en los que no es posible aprender con los modelos tradicionales y la distancia social prima regulando las actividades y conductas de todas las personas, la manera más adecuada de permitir la continuidad del aprendizaje es por medio de la innovación educativa (Moreno, 2020), y las TIC y, en particular, las tecnologías móviles son la respuesta más efectiva.

Los profesores y estudiantes cuentan con una lista robusta de aplicaciones móviles que se ofrecen con fines educativos y en las que, si no cuentan con un instrumento que permita verificar el valor educativo de sus contenidos, es posible que inviertan tiempo y recursos sin lograr sus objetivos de aprendizaje (Cherner et al., 2016; Papadakis et al., 2017). Si bien en la actualidad existen algunos instrumentos que permiten evaluar si una aplicación móvil tiene valor educativo, se observó la necesidad de contar con una rúbrica actualizada (Cherner et al., 2016) y contextualizada a la realidad del sistema educativo chileno y latino hispanohablante.

La estructura de la rúbrica integral para evaluar aplicaciones educativas de Cherner et al. (2016) ha sido destacada en estudios científicos por permitir observar un amplio espectro de aplicaciones móviles sin restricciones, y ha servido de referente para diseñar RubricApp. El proceso de adaptación de la rúbrica integral contempla el cambio idiomático de inglés a español y los ajustes de los indicadores para su contextualización. El juicio de los expertos es la instancia que permite mayor precisión en cuanto a los ajustes de lenguaje y para asegurar la coherencia y validez de los descriptores y las dimensiones (Escobar-Pérez y Cuervo-Martínez, 2008). La opinión del comité de expertos arroja como resultado una rúbrica con 3 dimensiones que totaliza 10 indicadores. Dichas dimensiones permiten observar el valor pedagógico, el diseño, y la participación y motivación de una aplicación móvil. Sin duda, como herramienta de observación de la calidad de las aplicaciones móviles, RubricApp será un aporte significativo para profesores, educadoras de párvulos, padres y apoderados, incluso estudiantes de niveles de Segundaria a Pregrado, incluyendo a los propios desarrolladores de dichas aplicaciones educativas, quienes contarán con una rúbrica que reduce tiempos de evaluación en relación con otros instrumentos disponibles, que tienen varios indicadores y se debe invertir mucho tiempo al momento de evaluar una aplicación móvil.

Más significativo aún es contar con una rúbrica que facilite la elección de aplicaciones educativas en los distintos niveles de enseñanza, teniendo en consideración que el contexto de pandemia en el que se vive desde febrero de 2020 ha impactado en la educación y ha causado la repentina transformación de los procesos de enseñanza a formatos completamente en línea. Como señala Daruwala (2020), en este escenario, la mayoría de la gente estaría de acuerdo en que tener tecnología durante esta pandemia es preferible a no tener posibilidad de contacto con otros, de ahí la importancia de contar con aplicaciones educativas, plataformas y recursos que puedan contribuir para facilitar el aprendizaje de los estudiantes y proporcionar atención social e interacción durante este período. En este contexto, la Unesco (2020) sugiere un listado de recursos, la mayoría son soluciones gratuitas y muchas se adaptan a varios idiomas. Si bien estas soluciones no cuentan con su respaldo explícito, tienden a tener un amplio alcance, una sólida base de usuarios y una evidencia de impacto, se clasifican en función de las necesidades de aprendizaje a distancia, pero la mayoría ofrecen funcionalidades en múltiples categorías. Todo esto, sin duda, nos lleva a considerar que el impacto en los aprendizajes de los estudiantes a través del uso de estas aplicaciones está supeditado, entre otros factores, al valor educativo de los recursos que se les sugiere para su aprendizaje, lo que releva el objetivo de este artículo y la propuesta de contar con una rúbrica que facilite la toma de decisiones por parte no solo de docentes, sino también de padres y apoderados.

El instrumento queda a disposición gratuita solicitándolo al correo electrónico de la autora principal.

\section{Limitaciones}

En cuanto a las limitaciones de RubricApp estamos de acuerdo con los autores de la rúbrica integral de referencia de nuestro trabajo, quienes sostienen que el acelerado desarrollo de las aplicaciones móviles conlleva a la necesidad de revisar y modificar los indicadores del instrumento (Cherner et al, 2016). Es posible que, en los próximos años, los avances tecnológicos permitan incorporar otros elementos o tecnologías en 
el diseño de las aplicaciones móviles, por tanto, es recomendable prestar atención a las dimensiones del instrumento, siendo posible agregar, eliminar o ajustar indicadores y descriptores de desempeño.

Esta rúbrica ofrece una visión global de aspectos pedagógicos, diseño y motivaciones para su uso, y permite a un profesor apreciar el valor educativo de una aplicación móvil. Sin embargo, se advierte que hay limitaciones para evaluar las especificidades de las disciplinas, por ejemplo, lenguaje, literatura, matemáticas, ciencias o artes, por mencionar algunas, ya que no orienta sobre el valor pedagógico específico de sus disciplinas. Para un fin de esas características sería necesario adecuar los indicadores y los respectivos descriptores.

RubricApp es un instrumento que orienta el criterio pedagógico de un profesor en cuanto al valor educativo de una aplicación móvil, es importante señalar que, por sí misma, no garantiza un efecto en el aprendizaje del estudiante. El fenómeno del aprendizaje es multifactorial, no obstante, la evidencia empírica acumulada en relación con la integración de las TIC y, en particular, del aprendizaje móvil, confirman la imperiosa necesidad de planificar el uso de la tecnología vinculando a objetivos de aprendizajes (Hernández-Carmelo et al., 2018), que solo un profesor puede determinar observando las características y las necesidades de sus estudiantes.

El estudio se realiza con profesores chilenos de niveles de Preescolar y Primaria, por lo tanto, los resultados compartidos dan cuenta de un instrumento apropiado para estos niveles de enseñanza, que es interesante ampliar a docentes de niveles superiores.

\section{Proyecciones}

Se ha iniciado un proyecto piloto de capacitación en el uso de las herramientas TIC y las aplicaciones móviles para educadores de niveles de primera infancia, quienes, de manera voluntaria, utilizan RubricApp para evaluar aplicaciones educativas dirigidas a niños del nivel Preescolar y de Primaria. Con los resultados de dichas evaluaciones se espera disponer de un catálogo de las mejores aplicaciones educativas en idioma español en los establecimientos públicos de la región de Bío-Bío en Chile, que pueda servir de apoyo a los procesos de enseñanza-aprendizaje, especialmente, en estos tiempos de pandemia.

Se espera avanzar en estudios futuros en la aplicación de RubricApp con profesores de países de Latinoamérica, que permita observar, en otra población, las cualidades del instrumento y su utilidad en otros contextos educativos.

\section{Financiamiento}

Este trabajo ha sido desarrollado gracias al financiamiento otorgado por la Agencia Nacional de Investigación y Desarrollo de Chile (ANID) / Programa Beca Doctorado Nacional /2017- 21171845. Doctorado en
Educación en Consorcio Universidad Católica de la Santísima Concepción (UCSC). Un especial agradecimiento al Centro de Investigación en Educación y Desarrollo, CIEDE-UCSC, financiamiento otorgado al: Proyecto CIEDE 02/2018, Investigador responsable: Dra. María Graciela Badilla Quintana.

\section{Declaración de divulgación autores}

No existen intereses en conflicto.

\section{Referencias}

Angell, K. (2015). The application of reliability and validity measures to assess the effectiveness of an undergraduate citation rubric. Behavioral \& Social Sciences Librarian, 34 (1), 2-15. https://doi.org/10.10 80/01639269.2015.996481

Aprender Jugando (2019). Sílabas Leon, aprender a leer con sílabas (22) [Aplicación móvil]. GooglePlay. https://bit.ly/2FOdbmA

Aznar-Díaz, I., Cáceres-Reche, M., Trujillo-Torre, J. y Romero-Rodríguez, J. (2019). Mobile learning y tecnologías móviles emergentes en Educación Infantil: percepciones de los maestros en formación. Revista Espacios, 40(5), 14. http://www.revistaespacios.com/ a19v40n05/a19v40n05p14.pdf

Badia, G. (2019). Holistic or analytic rubrics? Grading Information literacy instruction. College \& Undergaduate Libraries. https://doi.org/10.1080/10691316.2 019.1638081

Besoli, G., Palomas, N. y Chamarro, A. (2018). Uso del móvil en padres, niños y adolescentes: Creencias acerca de sus riesgos y beneficios. Aloma Revista de Psicología, Ciències de l’Educació i de l'Esport, 36(1), 29-39. https://bit.ly/2XS3x7s

Bonneton-Botté, N., Fleury, S., Girard, N., Le Magadou, M., Cherbonnier, A., Renault, M., Anquetil, E. y Jamet, E. (2020). Can tablet apps support the learning of handwriting? An investigation of learning outcomes in kindergarten classroom. Computer \& Education, 151. https://doi.org/10.1016/j.compedu.2020.103831

Buckler, T. y Peterson, M. (2012). Is there an App for that? Developing an evaluation rubric for Apps for use with adults with special needs. The Journal of BSN Honors Research, 5(1), 19-32. https://bit.ly/36NgjIo

Carrion, C. C. (2019). Aprender a leer (5.1.5) [Aplicación móvil]. GooglePlay. https://bit.ly/2Hn9aGz

Cherner, T., Lee, C.Y., Fegely, A. y Santaniello, L. (2016). A detailed rubric for assessing the quality of teacher resource apps. Journal of information technology education: innovations in practice, 15, 117-143. http://www.informingscience.org/Publications/3527

Clement, J. (28 de septiembre de 2020). Number of apps available in leading app stores 2020. https://bit. ly/2LZtVGQ

Daruwala, N. (2020). Generation Lockdown: Exploring possible predictors of technology phobia during the Coronavirus self-isolation period. Aloma, 38(1), 1520. https://bit.ly/30vS9jE 
Educaplanet. (2019). Aprender a leer con Grin (3.4.9) [Aplicación móvil]. GooglePlay. https://bit.ly/ 2Thl476

Escobar-Pérez, J. y Cuervo-Martínez, A. (2008). Validez de contenido y juicio de expertos: una aproximación a su utilización. Avances en Medición, 6, 27-36. https:// bit.ly/37d9imF

Faizal-Yamimi, M., Abdul-Rahim, S., Faruk, M., Ghazali, B. y Lokman-Hafiz, A. (2018). Literature Review of Educational App Evaluation Rubrics. [conferencia]. E-proceedings LSP-GABC 201811 (1), 103-108. https://bit.ly/3ewjRTi

Fuentes, J.L., Albertos, J. y Torrano, F. (2019). Hacia el Mobile-Learning en la escuela: análisis de factores críticos en el uso de las tablets en centros educativos españoles. Education in the Knowledge Society (EKS), 20, 3-17. https://doi.org/10.14201/eks2019_20_a3

García-Sanz, M. (2014). La evaluación de competencias en Educación Superior mediante rúbricas un caso práctico. Revista Electrónica Interuniversitaria de Formación de Profesorado, 17(1), 87-106. http://dx.doi. org/10.6018/reifop.17.1. 198861

Gil-Quintana, J. (2019). Interconectados apostando por la construcción colectiva del conocimiento. Aprendizaje móvil en educación infantil y primaria. PíxelBIT Revista de Medios y Educación, 54, 185-203. https:// doi.org/10.12795/pixelbit.2019.i54.10

Gillate, I., Vicent, N., Gómez, C. y Marín, S. (2017). Características y dimensiones educativas en apps de educación patrimonial. Análisis a partir del método OEPE. Estudios Pedagógicos, 4, 115-136. https://doi. org/10.4067/S0718-07052017000400006

Gómez-Díaz, R., García-Rodríguez, A. y Cordón-García, J. (2015). APPrender a leer y escribir: aplicaciones para el aprendizaje de la lectoescritura. Education in the Knowledge Society (EKS), 16(4), 118-137. http:// dx.doi.org/10.14201/eks2015164118137

Gonzaga, L. (2016). Más allá de la calificación instrumentos para evaluar el aprendizaje. Universidad de Concepción. https://bit.ly/3p3rlCj

Hernández-Carmelo, G., Trujillo-Torres, J., Cáceres -Reche, M. y Soler-Costa, M. (2018). Using and integration of ICT in a diverse educational context of Santander (Colombia). Journal of Technology and Science Education JOTSE, 8(4), 254-267. http://dx. doi.org/10.3926/jotse.314

Ifinedo, E., Rikala, J. y Hämälïnen, T. (2019). Factors affecting Nigeria teacher educators' technology integration: considering characteristics, knowledge constructs, ICT practices and beliefs. Computers \& Education, 146, 103760. https://doi.org/10.1016/j. compedu.2019.103760.

Jescovitch, L., Scott, E., Cerchiara, J., Doherty, J., Wenderoth, M., Merrill, J., Urban-Lurain, M., y Haudek, K. (2019). Deconstruction of Holistic Rubrics into Analytic Rubrics for Large-Scale Assessments of Students' Reasoning of Complex Science Concepts, Practical Assessment, Research, and Evaluation. 24 https://doi.org/10.7275/9h7f-mp76 Available at: https://scholarworks.umass.edu/pare/vol24/iss1/7
Khanna, A. (01 de abril de 2020). Stats for mobile app download and usage in 2020. https://bit.ly/36qOOnO Kortabitarte, A., Gillate, I., Luna, U. e Ibáñez-Etxeberria, A. (2018). Las aplicaciones móviles como recurso de apoyo en el aula de ciencias sociales: estudio exploratorio con la app "Architecture Gothique/Romane" en educación secundaria. ENSAYOS, Revista de la Facultad de Albacete, 33 (1), 65-79. https://revista. uclm.es/index.php/ensayos/article/view/1743

Lee, Ch.-Y. y Cherner, T. -S. (2015). A comprehensive evaluation rubric for assessing instructional apps. Journal of information technology education: research, 14. https://bit.ly/2yv87A1

https://doi.org/10.1177/1053451215589179

Mize, M., Park, Y., Schramm-Possinger, M. y Coleman, M. (2019). Developing a rubric for Learners with reading difficulties. Intervention in School and Clinic, 55(3), 145-153. https://journals.sagepub.com/ doi/10.1177/1053451219842237

Moreno-Correa, S. (2020). La innovación educativa en los tiempos del Coronavirus. Salutem Scientia Spiritus, 6(1),14-26. https://bit.ly/36VFpF9

Nikolopoulou, K. (2020). Secondary education teachers? Perceptions of mobile phone and tablet use in classrooms: benefits, constraints and concerns. Journal of Computers in Education, 7(2), 257-275. https://doi.org/10.1007/s40692-020-00156-7

Ok, M., Kim, M., Kang, E. y Bryant, B. (2016). How to find good apps: An evaluation rubric for instructional apps for teaching students with learning disabilities. Journal Intervention in School and Clinic (ISC), 51 (4) 244-252.https://doi.org/10.1177/ 1053451215589179

Panadero, E. \& Jonsson, A. (2020). A critical review of the arguments against the use of rubrics. Educational Research Review. 30. https://doi.org/10.1016/j.edurev. 2020.100329

Papadakis, S., Kalagiannakis, M. y Zanaris, N. (2017). Designing and creating an educational app rubric for preschool teachers. Education and Information Technologies, 22, 3147-3165. https://doi.org/10.1007/ s10639-017-9579-0

ParKel Soft - Apps for School \& Kindergarten Kids. (2020). Letrakid Aprende a Escribir para Niños. (1.9.3) [Aplicación móvil]. GooglePlay. https://bit.ly/ 2HEqO8O

Parsons, D. y MacCallum, K. (2017). A learning theory rubric for evaluating mobile learning activities. International Journal of Online Pedagogy and Course Design, 7(4). https://doi.org/10.4018/IJOPCD.2017100102

Ricoy, M. y Sánchez-Martinez, C. (2019). Tablet use in primary Education. Technology, Pedagogy and Education, 28(3), 301-316. https://doi.org/10.1080/14759 39X.2019.1608291

Salem, N. y Mohammadzadeh, B. (2018). A study on the integration of ICT by EFL Teachers in Libya. EURASIA Journal of Mathematics, Science and Technology Education, 14(7), 2787-2801. https://doi.org/ 10.29333/ejmste/90594 
Santiago, R., Trabaldo, S., Kamijo, M. y Fernández, A. 2015). Mobile learning nuevas realidades en el aula. Grupo océano. https://bit.ly/3gFc5ao

Semerci, A. y Aydin, M. (2018). Examining high school teachers' attitudes towards ICT use in education. International Journal of Progressive Education, 14(2), 93.105.https://doi.org/10.29329/ijpe.2018.139.7

SensorTower. (2020, 7 de febrero). Top Apps worldwide for January 2020 by downloads. https://bit.ly/ 3ghdQKF

Statista. (2020). Number of available apps in the Apple App Store from $1^{\text {st }}$ quarter 2020. https://bit.ly/3cZMQxt Stein, H., Gurevich, I. y Gorev, D. (2019). Integration of technology by novice mathematics teachers - what facilitates such integration and what makes it difficult? Education and Information Technologies, 25, 141161. https://doi.org/10.1007/s10639-019-09950-y

Tondeur, J., Pareja-Roblin, N., Van Braak, J., Voogt, J. y Prestridge, S. (2016). Preparaing beginning teachers for technology integration in Education: ready for take-off? Technology, Pedagogy and Education, 26(2), 157-177. https://doi.org/10.1080/1475939X.2016. 1193556

Torres Díaz, J.C., Infante Moro, A. y Torres Carrión, P. (2015). Aprendizaje móvil: perspectivas. RUSC. Universities and Knowledge Society Journal, 12(1), 38-49. http://dx.doi.org/10.7238/rusc.v12i1.1944

Unesco. (2020). Distance learning solutions. https:// en.unesco.org/covid19/educationresponse/solutions

Weng, P. (2015). Developing an app evaluation rubric for practitioners in special education. Journal of Special Education Technology, 30 (1), 43-58. https:// doi.org/10.1177/016264341503000104

Zaranis, N. y Oikonomidis, V. (2014). The main factors of the attitudes of Greek kindergarten teachers towards information and communication technology. European Early Childhoood Education Research Journal, 24(4), 615-632. http://dx.doi.org/10.1080/1 350293X.2014.970853 
Anexo 1. Resultados RubricApp

\begin{tabular}{|c|c|c|c|c|c|c|c|c|c|}
\hline \multirow[t]{2}{*}{ Nombre Apps } & \multirow[t]{2}{*}{ SO } & \multirow[t]{2}{*}{ MB } & \multirow[t]{2}{*}{ Precio } & \multirow[t]{2}{*}{ Edad } & \multirow{2}{*}{$\begin{array}{l}\text { Habilidades } \\
\text { Aprendizajes }\end{array}$} & \multirow[t]{2}{*}{ Descripción } & \multicolumn{3}{|c|}{ Resultados RubricApp } \\
\hline & & & & & & & \multicolumn{2}{|c|}{ Dimensión } & \% Logro \\
\hline \multirow{3}{*}{$\begin{array}{l}\text { Sílabas León, aprender } \\
\text { a leer con sílabas }{ }^{1}\end{array}$} & \multirow[t]{3}{*}{ Android } & \multirow[t]{3}{*}{32} & \multirow[t]{3}{*}{ Gratis } & \multirow[t]{3}{*}{6 a 12 años } & \multirow[t]{3}{*}{ Reconocer letras y sílabas } & \multirow{3}{*}{$\begin{array}{l}\text { Aprendizaje de sílabas en } \\
4 \text { ambientes lúdicos: Paso } \\
\text { a paso, } \\
\text { Silabas locas, Cuenta sílabas } \\
\text { e } \\
\text { Intruso. Cada etapa les } \\
\text { permite construir sílabas } \\
\text { y palabras. }\end{array}$} & VP & 11 & $73 \%$ \\
\hline & & & & & & & $\mathrm{D}$ & 12 & $80 \%$ \\
\hline & & & & & & & PM & 13 & $65 \%$ \\
\hline \multicolumn{7}{|c|}{ Resultado global de RubricApp para la aplicación móvil Sílabas León. Valor educativo alto } & Total & 36 & \\
\hline \multirow[t]{2}{*}{ Aprender a leer ${ }^{2}$} & \multirow[t]{2}{*}{ Android } & \multirow[t]{2}{*}{39} & \multirow[t]{2}{*}{ Gratis } & \multirow[t]{2}{*}{ Hasta 5 años } & \multirow{2}{*}{$\begin{array}{l}\text { Reconocer sílabas } \\
\text { Formar sílabas y palabras } \\
\text { Leer palabras y frases cortas } \\
\text { Aprender a escribir } \\
\text { Vocabulario }\end{array}$} & \multirow{2}{*}{$\begin{array}{l}\text { Aprendizaje de sílabas } \\
\text { y vocabulario. }\end{array}$} & VP & 11 & $73 \%$ \\
\hline & & & & & & & $\overline{\mathrm{PM}}$ & 11 & $70 \%$ \\
\hline \multicolumn{7}{|c|}{ Resultado global de RubricApp para la aplicación móvil Aprender a leer: Valor educativo bajo } & Total & 26 & \\
\hline \multirow{3}{*}{$\begin{array}{l}\text { Leo con Grin: aprender } \\
\text { a leer }^{3} \\
\text { APRENDER }\end{array}$} & \multirow[t]{3}{*}{ Android } & \multirow[t]{3}{*}{51} & \multirow[t]{3}{*}{ Gratis } & \multirow[t]{3}{*}{ Hasta 8 años } & \multirow{3}{*}{$\begin{array}{l}\text { Memorización visual } \\
\text { y auditiva. } \\
\text { Identificación y asociación. } \\
\text { Discriminación. } \\
\text { Comprensión } \\
\text { Lectoescritura }\end{array}$} & \multirow[t]{3}{*}{$\begin{array}{l}\text { Aprendizajes de letras } \\
\text { en distintos niveles. }\end{array}$} & VP & 9 & $60 \%$ \\
\hline & & & & & & & $\overline{\mathrm{D}}$ & 10 & $66 \%$ \\
\hline & & & & & & & PM & 9 & $45 \%$ \\
\hline \multicolumn{7}{|c|}{ Resultado Global de RubricApp para aplicación móvil Leo con Grin. Valor educativo medio } & Total & 28 & \\
\hline
\end{tabular}

Nota. Elaboración propia.

1. Aplicación y logo autoría de Aprender Jugando. (2019). Sílabas León, aprender a leer con sílabas (22) [Aplicación móvil]. Google Play. https://bit.ly/2FOdbmA

2. Aplicación y logo autoría de Carrion Castillo, C\&C. (2019). Aprender a leer (5.1.5) [Aplicación móvil]. Google Play. https://bit.ly/2Hn9aGz

3. Educaplanet. (2019). Leo con Grin (3.4.9) [Aplicación móvil]. Google Play. https://bit.ly/2Thl476 